\title{
Isolated Tumor Cells and Long-Term Prognosis of Patients with Melanoma
}

\author{
A. C. J. van Akkooi, MSc, J. H. W. de Wilt, MD, PhD, C. Verhoef, MD, \\ and A. M. M. Eggermont, MD, PhD
}

Surgical Oncology, Erasmus University Medical Center-Daniel den Hoed Cancer Center, Groene Hilledijk 301, Kamer A1-41, Rotterdam, 3075 EA, Netherlands

To the Editor:

Sheri et al. reported in the Annals of Surgical Oncology on the significance of isolated tumor cells (ITC). ${ }^{1}$ At the moment the subject of ITC or SUBmicrometastases, minimal sentinel node (SN) tumor burden is a hot issue. Therefore, it is very interesting that the experience from the John Wayne Cancer Institute (JWCI) has been reported. This recent publication does raise a few questions with regard to the different results acquired by the authors compared with recent publications by others. ${ }^{2,3}$

The first point of note is that the experience from the JWCI goes back to 1991; this is an advantage, because this leads to a longer median follow-up compared with other single-center studies. ${ }^{2,3}$ However, at the same time, it means that patients from the early days of SN staging have been included. This is a drawback because the pathology protocol, which nowadays includes bivalving through the hilum, more step-sections, more immunostains, and smaller intervals, ${ }^{4,5}$ was less extensive in the early days of SN staging. This may be reflected in the very low SN positivity identification rate of only $15 \%$, compared with many other studies, which report rates between $20 \%$ and $30 \%$. $^{5,6}$

This is supported by the fact that the authors have found additional positive nodes in the completion lymph node dissection (CLND) in 6 of 52 patients

Published online January 15, 2008

Address correspondence and reprint requests to: A. C. J. van Akkooi, MSc; E-mail: a.vanakkooi@erasmusmc.nl

Published by Springer Science+Business Media, LLC $\odot$ The Authors(s) 2008
(12\%) with ITC. An extensive "modern" pathology protocol should probably have picked up larger tumor lesions in the original $\mathrm{SN}$. We wonder if the authors have extended the workup of the SNs of these six patients to meet the standard extensive workup used nowadays?

The second point of note is the extremely good survival of SN negative patients reported by the authors of $94 \%$ at $5 \mathrm{y}$ ears. This is somewhat higher than in most other series, including the MSLT-I trial, which report rates of around 90\%., ${ }^{6,7}$ The reason for this is that the $\mathrm{SN}$ negative patients had a much smaller median Breslow thickness $(1.2 \mathrm{~mm}$ !) compared with the MSLT-I trial $(1.8 \mathrm{~mm})$ or other series. ${ }^{5,7}$

The authors report that even if ITC would have implications, its occurrence is very low at only $4 \%$. However, shouldn't this rate be calculated as proportion of all SN positive patients? This is 57/ $214=27 \%$, who could possibly not benefit from CLND in our experience.

We believe that isolated tumor cells are not always important, as has recently been demonstrated in a multicenter trial for the detection of isolated tumor cells using polymerase chain reaction (PCR) techniques. Scoggins et al. did not demonstrate additional prognostic information beyond standard hematoxylin-eosin (HE) staining of SNs. ${ }^{8}$ Although Scheri et al. demonstrate a decreased survival in their group of patients with ITC defined as $<0.2 \mathrm{~mm}$, ${ }^{1}$ we believe that the prognosis of patients with SUB-micrometastatic disease as defined as $<0.1 \mathrm{~mm}$ has no impact on overall survival. A multicenter study conducted within the EORTC 
Melanoma Group and recently presented at ECCO 14 in Barcelona, showed that patients with $<0.1 \mathrm{~mm}$ SUB-micrometastases had an excellent 5 -year overall survival rate of $91 \%$. The rate of additional nonSN positivity in CLND was 3\% (which is identical to the false negative rate in the MSLT- 1 trial). ${ }^{9}$ We have also addressed the limit of $0.2 \mathrm{~mm}$ as definition for SUB-microscopic disease. The overall survival decreased slightly to $89 \%$ at $5 \mathrm{y}$ ears; however the occurrence of CLND positivity increases significantly to $10 \%$, which leads us to believe that melanoma and breast cancer have a different biology and thus require different cut-off values for SUBmicrometastases.

Whether the excellent survival of patients with $<0.1 \mathrm{~mm}$ SUB-micrometastatic disease is achieved because of or in spite of CLND remains to be seen and is the subject of an EORTC Melanoma Group registration study, the MINITUB, which shall be activated within the next year. Perhaps the MSLT-II trial will also answer the question of which group(s) of SN patients may or may not benefit from CLND. Until more data are available, clinicians should realize that advising removal of all regional nodes to all of their patients might change with respect to further insights in long-term behavior of SUBmicrometastatic involvement of the SN.

\section{OPEN ACCESS}

This article is distributed under the terms of the Creative Commons Attribution Noncommercial License which permits any noncommercial use, distribution, and reproduction in any medium, provided the original author(s) and source are credited.

\section{REFERENCES}

1. Scheri RP, Essner R, Turner RR, et al. Isolated tumor cells in the sentinel node affect long-term prognosis of patients with melanoma. Ann Surg Oncol 2007; 14:2861-6.

2. van Akkooi AC, de Wilt JH, Verhoef C, et al. Clinical relevance of melanoma micrometastases $(<0.1 \mathrm{~mm})$ in sentinel nodes: are these nodes to be considered negative?. Ann Oncol 2006; 17:1578-85.

3. Govindarajan A, Ghazarian DM, McCready DR, et al. Histological features of melanoma sentinel lymph node metastases associated with status of the completion lymphadenectomy and rate of subsequent relapse. Ann Surg Oncol 2007; 14:906-12.

4. Cook MG, Green MA, Anderson B, et al. The development of optimal pathological assessment of sentinel lymph nodes for melanoma. J Pathol 2003; 200:314-9.

5. van Akkooi AC, de Wilt JH, Verhoef C, et al. High positive sentinel node identification rate by EORTC melanoma group protocol. Prognostic indicators of metastatic patterns after sentinel node biopsy in melanoma. Eur J Cancer 2006; 42:37280 .

6. Estourgie $\mathrm{SH}$, Nieweg OE, Valdes Olmos RA, et al. Review and evaluation of sentinel node procedures in 250 melanoma patients with a median follow-up of 6y ears. Ann Surg Oncol 2003; 10:681-8.

7. Morton DL, Thompson JF, Cochran AJ, et al. Sentinel-node biopsy or nodal observation in melanoma. N Engl J Med 2006; 355:1307-17.

8. Scoggins CR, Ross MI, Reintgen DS, et al. Prospective multiinstitutional study of reverse transcriptase polymerase chain reaction for molecular staging of melanoma. J Clin Oncol 2006; 24:2849-57.

9. van Akkooi AC, Nowecki ZI, Voit C, Schaefer G, Michej W, Kliffen M, Schmitz PIM, Ruka W, Eggermont AMM.. Prognosis depends on micro-anatomic patterns of melanoma micrometastases within the sentinel node (SN). A multicenter study in 388 SN positive patients. Eur $J$ Cancer Supplements 2007; 5:397; ; abstract 7006 . 